\title{
NCRA: An Opportunistic Networks Routing Algorithm based on Network Coding
}

\author{
Hongfeng Wang ${ }^{1}$, Dingding Zhou ${ }^{3}$ and Shi Dong ${ }^{1,2}$ \\ ${ }^{1}$ School of Computer Science and Technology, Zhoukou Normal University, China, \\ Zhoukou, 466001, China \\ ${ }^{2}$ School of Computer Science and Technology, Huazhong University of Science \\ and \\ Technology, Wuhan, China \\ njbsok@gmail.com \\ ${ }^{3}$ Laboratory and Equipment Management Department, Zhoukou Normal \\ University, China
}

\begin{abstract}
Opportunistic network realizes the communication between nodes by "storage-carryforward" mode, the traditional routing algorithms can not be applied to the opportunistic network, to address the problems in opportunistic networks that nodes employing routing algorithms based on epidemic mechanism can't sense their neighboring nodes timely and many redundant data packets permeate through the network. This article proposes network routing algorithm based on an efficient opportunistic network. The algorithm is based on network coding method to solve routing problems, compared with existing routing method. NCRA method can handle dynamic route to improve the transmission success rate and reduce the transmission delay, reduce the routing overhead and short the buffer time, decrease the hops of overall opportunistic network.
\end{abstract}

Keywords: Opportunistic network; network coding; routing overhead; transmission delay; transmission success rate.

\section{Introduction}

Nowadays, some of the concepts of the opportunistic network come from the early delay tolerant network (DTN, Delay Tolerant Network)[6].It can be viewed as a general DTN network characteristics with wireless ad hoc networks, but there is no uniform definition.[5]proposes a descriptive definition by analysis of the opportunistic network characteristics, the opportunistic network is a self-organizing network communication which do not need to own the full path from the source to destination nodes, it uses node moving encounter opportunities to finish communication. In the traditional ad hoc network, the network is mostly connected. Between any node pair there is at least one complete end side communication path. For opportunistic networks, at a particular moment, the network may be divided into sub-regions that can not communicate. In order to better understand the concept of the opportunistic network, through a schematic analysis as shown in Figure 1, at the time t1, S and R is located in a different connectivity domain without communication path, Therefore, $S$ will first send a message to a neighbor node 3 , since the node 3 has no proper opportunity to forward the message to the next hop node, which message in the local storage and waiting for a transmission opportunity, when the time $\mathrm{t} 2$ arrives, node 3 is moved into the communication range of the node 4 , and forwards the message to the node 4 , at time $\mathrm{t} 3$, the node 4 will transmit the message to the destination node $\mathrm{R}$, and the data transfer is completed. In order to solve the above problem, an opportunistic network routing algorithm based on network coding - NCRA is proposed in this paper. The method is compared with the traditional network routing 
algorithm, which has obvious improvement in the average transmission delay and transmission success rate, the average buffer time and routing overhead. The paper is organized as follows: Section 2 is a brief introduction of the opportunistic network routing algorithm; Section 3 analyzes the network coding mechanism, and proposed the mechanism-based routing algorithm; Section 4 evaluates the routing algorithm ; Section 5 is summary of the full article.

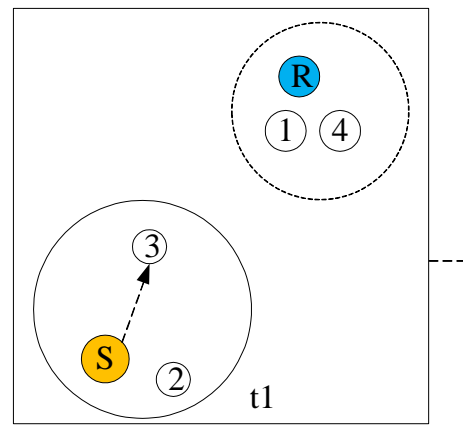

S Source node
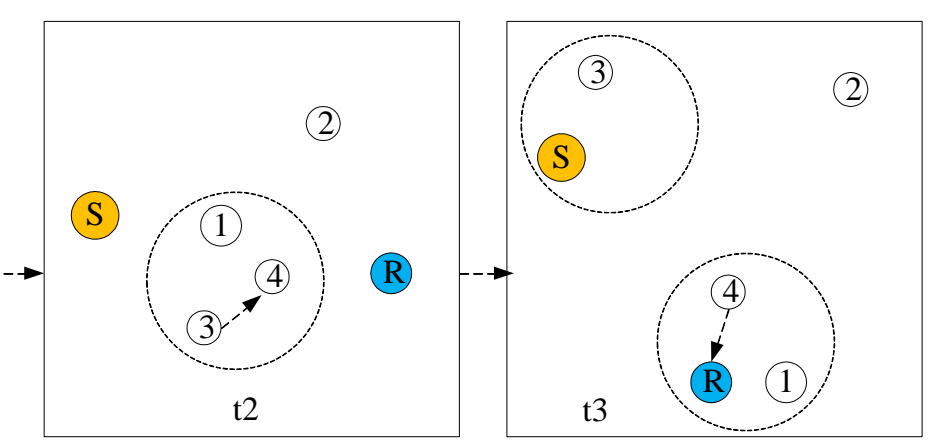

Communication range

Figure 1. Date Transfer in Opportunistic Networks

\section{Related Work}

First Contact, and Direct Delivery (also known as Direct Transmission) algorithm is based on the forwarding policy[8], the node of such routing algorithm in the during of packets transmission will not be copied, in the network, only one copy of the packets in the transmission. The direct delivery algorithms source node only in the face of the target node will be the message delivered to the next node, and First Contact algorithm source node message delivered to a node it encounters. Epidemic and Spray and Wait algorithm is based on the strategy of flooding in the class routing algorithm, when two nodes meet the other copy packets and limited packet lifetime or the number of copies to avoid excessive flooding. Epidemic algorithm idea is to exchange each other no packets. When two nodes meet enough swap, in theory, each of the non-isolated node will receive all messages, enabling the transmission of packets. The advantage of this algorithm is to maximize the success rate of packet transmission to reduce the transmission delay[10],the drawback is that there are a lot of a copy of the message in the network, it will consume a lot of network resources. Spray and Wait algorithm is divided into two stages: Spray stage, part of the message in the source node spread to neighboring nodes; stage of the Wait Spray, if spray stage did not find the destination node, then the node contains packets will transmit the packets to the destination node by way of Direct Delivery messages. The advantage of this algorithm is significantly less than Epidemic algorithm for the transmission amount; transmission delay is small and close to the optimal; it will have better scalability, no matter how the size of the network and node density change, which can have better performance[7, 9].

\section{Network Coding Mechanism}

\subsection{Network Coding}

Like many fundamental concepts, network coding is based on a simple basic idea which was first stated in its beautiful simplicity in the seminal paper by R. Ahlswede, 
N.Cai,[1]. The core notion of network coding is to allow and encourage mixing of data at intermediate network nodes. A receiver sees these data packets and deduces from them the messages that were originally intended for the data sink. In contrast to traditional ways to operate a network that try to avoid collisions of data streams as much as possible, this elegant principle implies a plethora of surprising results. One of the most exciting opportunities of the approach is the use of random mixing of data streams, thus freeing up the symmetrizing properties of random coding arguments in the analysis of networks. Not only is network coding a fresh and sharp tool that has the potential to open up stagnant fundamental areas of research, but due to its cross-cutting nature it naturally suggests a unified treatment of previously segmented areas. A striking example of the type of unification that network coding makes possible is the recently found elegant and complete characterization of the capacity of multicast networks, which was possible only through the joint treatment of coding and routing. In addition, Widmer et.al [12] introduces the network coding into the opportunistic networks and proposes opportunistic transmit mechanism based on random linear network coding, which maps different information source to a finite field, middle nodes use random linear coding vector to construct a new vector. When destination node obtains enough the information to decode the information vector, Transfer mechanism with network coding has great robustness and total number of information not tend to vary with the node density, so the total network can be better controlled. We will introduce theory related to network coding as follows.

\section{Max-flow Min-cut Theorem:}

Let $N=(V, E)$ be a network (directed graph) with $\mathrm{s}$ and $\mathrm{t}$ being the source and the sink of $\mathrm{N}$ respectively.

Maximum Flow's definition: The capacity of an edge is a mapping c : $\mathrm{E} \rightarrow \mathrm{R}+$, denoted by $\mathrm{c}_{\mathrm{uv}}$ or $\mathrm{c}(\mathrm{u}, \mathrm{v})$. It represents the maximum amount of flow that can pass through an edge. A flow is a mapping $\mathrm{f}$ : $\mathrm{ER}+$, denoted by $\mathrm{C}_{\mathrm{uv}}$ or $\mathrm{C}(\mathrm{u}, \mathrm{v})$, subject to the following two constraints:

1. Capacity Constraint:

$\forall(\mathrm{u}, \mathrm{v}) \in \mathrm{E}: \mathrm{f}_{\mathrm{uv}} \leqslant \mathrm{C}_{\mathrm{uv}}$

2. Conservation of Flows:

$\forall \mathrm{v} \in \mathrm{V} \backslash\{\mathrm{s}, \mathrm{t}\}: \sum_{\{\mathrm{u}:(\mathrm{u}, \mathrm{v}) \in \mathrm{E}\}} \mathrm{f}_{\mathrm{uv}}=\sum_{\{\mathrm{u}:(\mathrm{v}, \mathrm{u}) \in \mathrm{E}\}} \mathrm{f}_{\mathrm{vu}} \cdot$

The value of flow is defined by $|\mathrm{f}|=\sum_{\mathrm{v} \in \mathrm{V}} \mathrm{f}_{\mathrm{sv}}$, where $\mathrm{s}$ is the source of N. It represents the amount of flow passing from the source to the sink. Maximum Flow Problem: Maximize $|\mathrm{f}|$, that is, to route as much flow as possible from $\mathrm{s}$ to $\mathrm{t}$.

Minimum Cut's definition: An s-t cut $C=(S, T)$ is a partition of $V$ such that $s \in S$ and $t \in$ $\mathrm{T}$. The cut-set of $\mathrm{C}$ is the set $\{(\mathrm{u}, \mathrm{v}) \in \mathrm{E} \mathrm{u} \in \mathrm{S}, \mathrm{v} \in \mathrm{T}\}$. Note that if the edges in the cut-set of $\mathrm{C}$ are removed, $|\mathrm{f}|=0$. The capacity of an s-t cut is defined by $\mathrm{c}(\mathrm{S}, \mathrm{T})=\sum_{(\mathrm{u}, \mathrm{v}) \in \mathrm{S} \times \mathrm{T}} \mathrm{C}_{\mathrm{uv}}$.

Minimum s-t Cut Problem: Minimize c(S,T), that is, to determine $\mathrm{S}$ and $\mathrm{T}$ such that the capacity of the s-t cut is minimal. The maximum value of an s-t flow is equal to the minimum capacity over all s-t cuts. According to the randomness of the code coefficient generated, network coding can be divided into deterministic network coding and stochastic network coding, deterministic network coding needs to know the topology of the entire network, after the topological structure of network is known, coding vector should be pre-set. In this case, if network topology makes the change, for example node link suddenly is found disconnection, in addition, a new node joins or leaves, you will need to set the code vector. Therefore the cost and complexity of the network coding are very high, which is not suitable for complex and changeable wireless network environment. In order to solve the problem, T.Ho et al.[3] put forward the concept of random linear network coding, but this existed concept demands that the coding environment must be multicast and multi-source network environment, the coding method of data transmission and compression for distributed, but in a certain extent, it appears to 
broaden the application scenarios of network coding, therefore, the network coding is no longer limited to a fixed network topology. In the random linear network coding, the coding vector is based on the actual situation, it is key problem for the destination node whether can successfully decode the original data using random linear network coding. S.Jaggi[4]et.al prove that the finite domain of random access code vector is only big enough, at the destination node, success rate of the original data decoded that is close to 1 . But the more is finite domain, the greater is the additional overhead of nodes, so when the premise of the decoding success is satisfied, a smaller finite field should be selected. Chou PA[2] use random linear network coding and propose a scheme linear network coding for the real network, for random packet transmission delay may not be synchronized, loss possibility of each link packet is also unknown, network topology structure may be the dynamic changes. The scheme proposed uses a random linear network coding and random selects a finite field as the coding coefficients, which also does not need to know topology structure of network codec functions and network synchronization, the method has good robustness.

\subsection{Routing Algorithm based on Network Coding}

The section will propose the NCRA (routing algorithm based on network coding) algorithm, which adopts random linear network coding to decode the information of middle nodes. In random network coding, each information of node has a coding coefficient. Let source packets information is $p_{1}, p_{2}, \ldots, p_{n}$, then after packets information is decoded and is denoted as:

$$
D=\sum_{i=1}^{n} c_{i} p_{i}
$$

where $c_{i}$ represents coding coefficient, which is selected from finite field $\mathrm{F}_{\mathrm{q}}$, from overall view, when a node stores $p_{1}, p_{2}, \ldots, p_{n}$, meanwhile, some new packets also arrive, and those packets are expressed as $p_{\text {newl }}, p_{\text {new } 2}, \ldots, p_{\text {newn }}$, then after packets information is decoded and is denoted as:

$$
E=\sum_{i=1}^{n} c_{i} p_{i}+\sum_{j=1}^{m} c_{\text {newj }} p_{\text {newj }}
$$

In our method, the algorithm is divided by two processes. One is coding process, the other is decoding process. First, coding process: after network coding is applied into opportunistic networks, coding data packets transmitted between nodes contain two parts: respectively coding vector and the information vector, for not encoded node, coding vector is equal to the unit vector, it is added to code vectors in the source data package, the main purpose is that, when receiving end receives the data packet, which can successfully decode the original information. decoding process: The decoding process is divided into the same three steps: (1) intermediate nodes or the destination node message data extract corresponding coding information vector from the received encoded packets of information, in form of row vector into decoding matrix and prepare to decode. (2) Decoding will use Gauss elimination method[11] and is similar with the coding stage, if a message packets are found which has increased the rank of decoding matrix , then it will be as the new packet information, otherwise it is ignored. (3) On the basis of number theory's knowledge, if decoding success will be done at the destination node, you need to make the rank of decoding matrix which is equal to the number of data packet for source coding. 


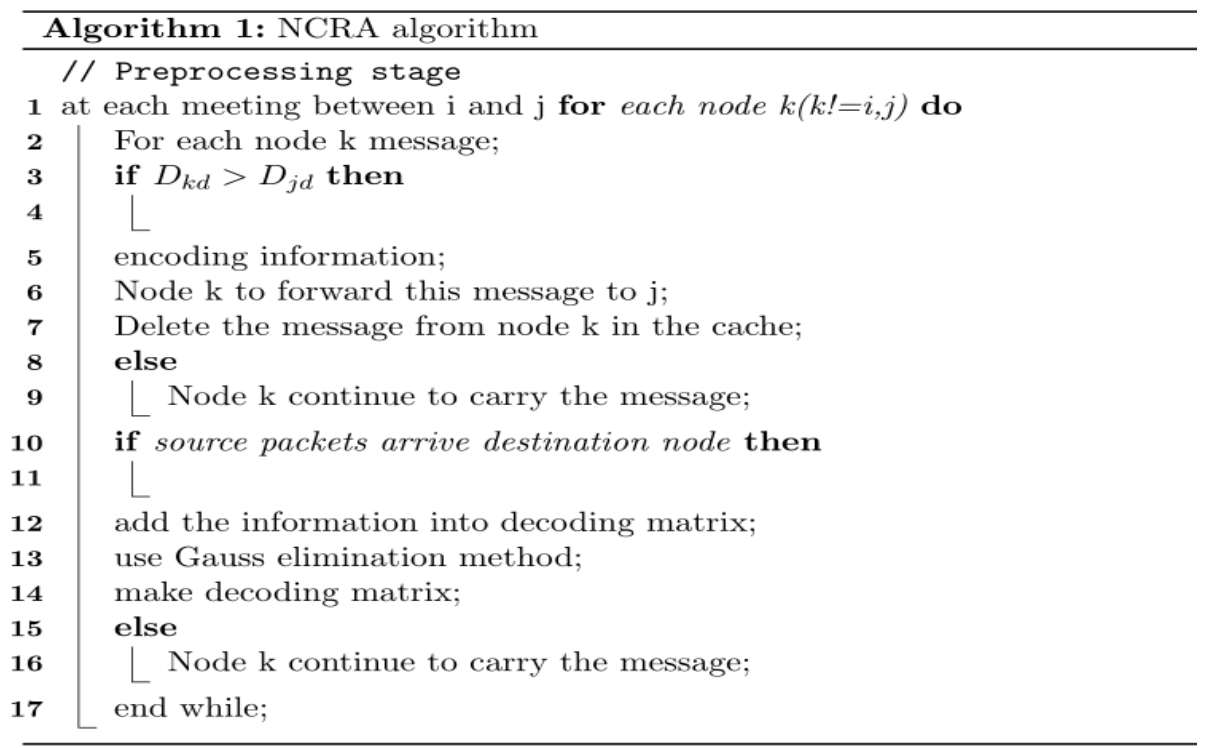

\section{Experiment Results}

\subsection{Experiment Environment}

The experiment uses ONE (the Opportunistic Networking Environment) simulation tools, and simulate pedestrian with smart Bluetooth devices walking in a real city scene, and analyzes the six kinds of routing algorithms. The specific scene is set as shown in Table 1. SPMBM (Shortest Path Map-Based Movement) model uses Dijkstra's algorithm to calculate the shortest path between the source and destination based on a map of the mid-point and path information, and the nodes along the path to move.

Table 1. Experiment Setup

\begin{tabular}{ll}
\hline Parameter & value \\
\hline Node number & 100 \\
Node movement speed & $2.5-8 \mathrm{Km} / \mathrm{h}$ \\
transmit ratio & 250 \\
node buffer & $5 \mathrm{MB}$ \\
simulate time & 12 hours \\
pps(k) & 69.2 \\
communication radius & $20 \mathrm{~m}$ \\
covering area & $5000 \mathrm{~m} * 5000 \mathrm{~m}$ \\
\hline
\end{tabular}

\subsection{Algorithm Evaluation and Analysis}

This article uses the following criteria to evaluate the performance of the routing algorithm:

(1)Transmission success rate

Transmission success rate (Delivery Ratio) is successfully in a certain period of time to reach the destination node the total number of packets and the source node sends the required transmission ratio of the total number of packets portrayed routing algorithm correctly forwarding packets to the destination node, is the most important of indicators.

(2)Transmission delay

Transmission delay (Delivery Delay) is the time required to reach the destination node from the source node in packets, usually using the average transmission delay to evaluate. Transmission delay is small means that the routing algorithm transmission capacity, high 
transmission efficiency, which means less network resources occupied during transmission.

(3)Routing overhead

Routing overhead refers to the total number of nodes forwarding packets within a certain time; usually use the ratio of the total number of packets reaches the destination node and forwarded message number to evaluate. If the routing overhead rate, which means nodes forwarding packets, a large number of flooded with a copy of the message in the network will increase the probability of packet collision, will be a large number of node energy consumption. This article considers the evaluation indicators of node energy consumption as an indirect indicator.

(4) Buffer time

Buffer time is the sum of packets each node cached time in its entire life cycle. The indicators describe a routing algorithm consumes nodes in the storage space, usually to evaluate the average caching time.

(5)The average number of hops

The average number of hops is the average value of the hop counts when message data are successfully transferred from a source node to the destination node.

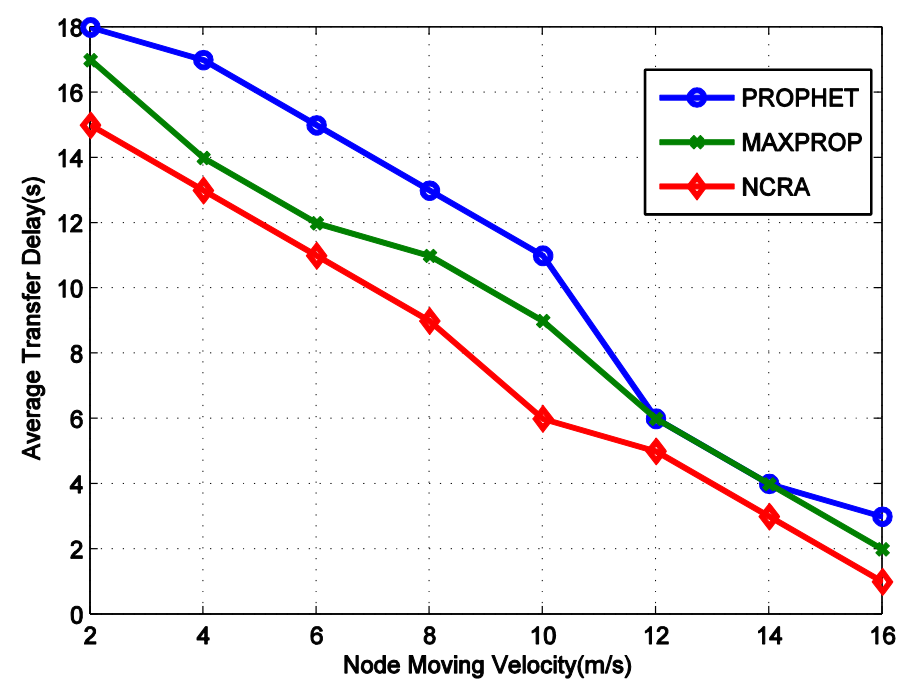

Figure 2. Average Transfer Delay of Node Buffer

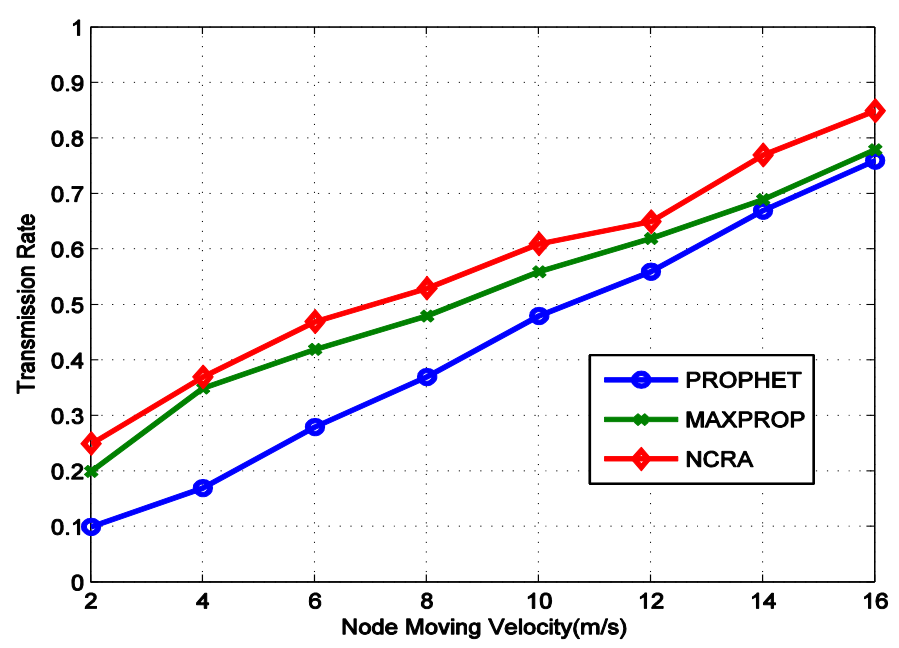

Figure 3. Transmission Rate of Node Buffer 


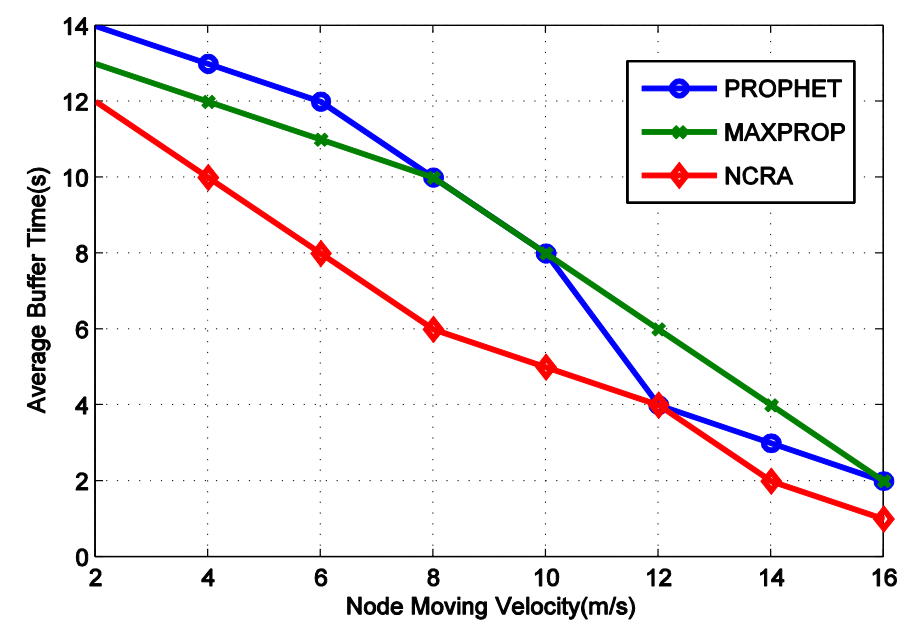

Figure 4. Average Buffer Time of Node Buffer

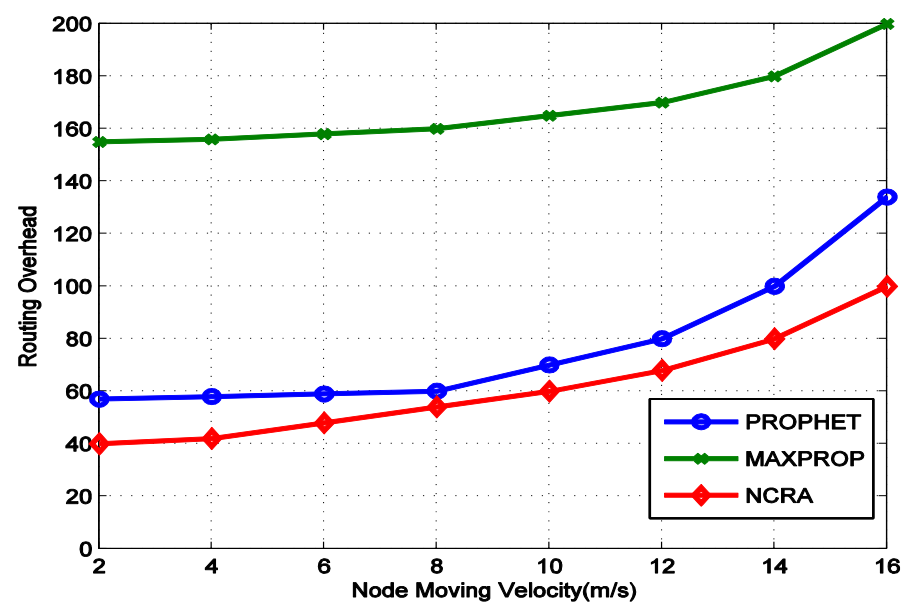

Figure 5. Routing Overhead of Node Buffer



Figure 6. Average Hops of Node Buffer 
Obviously, Figure 2 shows that a certain node cache transmission has certain effect to delay of the routing algorithm, and transmission delay increases as the node cache increases, average transmission delay of NCRA routing algorithm is the minimum, Figure 3-6 shows the relation between node cache and transmission success rate, the average cache time and routing overhead of each routing algorithm. The following conclusions can be drawn, 1, when node cache is the lower transmission success rate of the NCRA routing algorithm slightly is less than MAXPROP algorithm. With the increasing in the cache, NCRA algorithm will get the biggest transmission success rate. 2, as node caching increasing, the average buffer time of NCRA algorithm will be steady compared with several other routing algorithms, its average buffer time is minimum in four routing algorithm. 3, with the increasing in the node cache, routing overhead generated by the routing algorithm is constantly reduced, routing overhead of NCRA algorithm is minimal in the four algorithms, in addition, as shown in figure 6 , when node moving velocity is low, we find average hops number's increasing is faster as increasing of velocity compared to high velocity, and due to use network coding to improve the performance of transmission, so which will short the hops number. NCRC algorithm's hops is smallest compared to three routing algorithms.

\section{Conclusion}

The opportunistic network is kind of self-organizing network which do not need a complete link between the source node and the destination node, and take advantage of the node mobile encounter to communicate with each other. As a new construct the network method, the applications of opportunistic network is typically used in an environment that is tolerant of long delay and high error rate. Through a detailed analysis of the existing problems for the routing algorithm in opportunistic network, this paper proposes an efficient routing algorithm-NCRA. NCRA algorithm introduces network coding into routing forwarding process, which is different from traditional routing methods. The algorithm can effectively compensate for the defects of traditional routing algorithms. It can handle dynamic route to improve the transmission success rate and reduce the transmission delay, reduce the routing overhead and short the buffer time, decrease the hops of overall opportunistic network.

\section{Acknowledgements}

This paper is supported by Education Department of Henan Province Science and Technology Key Project Funding (14A520065), Research Innovation Foundation of Zhoukou Normal University (zknuA201408) and Introduction of Zhoukou Normal University scientific research grants project (ZKNU2014124), Scientific Research Fund of Henan Provincial Education Department of China(NO. 14B520057).

\section{References}

[1] R. Ahlswede, N. Cai, S.-Y. Li and R. W. Yeung, "Network information flow.Information Theory", IEEE Transactions on, vol.46, no.4, (2000), pp.1204-1216.

[2] P. A. Chou, Y. Wu and K. Jain, "Practical network coding", In Proceedings of the annual Allerton conference on communication control and computing, (2003); vol. 41, pp. 40-49.

[3] T. Ho, M. Médard, J. Shi, M. Effros and D. R. Karger, "On randomized network coding", In Proceedings of the Annual Allerton Conference on Communication Control and Computing, (2003), vol. 41, pp. 11-20.

[4] S. Jaggi, P. Sanders, P. A. Chou, M. Effros, S. Egner, K. Jain and L. M. Tolhuizen, "Polynomial time algorithms for multicast network code construction", Information Theory, IEEE Transactions on, vol.51, no.6, (2005), pp.1973-1982.

[5] H. Jun, M. H. Ammar and E. W. Zegura, "Power management in delay tolerant networks: A framework and knowledge-based mechanisms", In Proceedings of IEEE SECON, (2005), vol. 13, Citeseer. 
[6 A. Keränen, J. Ott and T. Kärkkäinen, “The one simulator for dtn protocol evaluation”, In Proceedings of the 2nd International Conference on Simulation Tools and Techniques, (2009); ICST (Institute for Computer Sciences, Social-Informatics and Telecommunications Engineering), pp. 55.

[7] G. Rong, T. Mei-si and J. Xiang-hong, "Spray and wait routing based on relay probability in dtn", Journal of Northeastern University (Natural Science), vol.12, (2012), 008.

[8] G. Sandulescu and S. Nadjm-Tehrani, "Opportunistic dtn routing with window aware adaptive replication", In Proceedings of the 4th Asian Conference on Internet Engineering, (2008); ACM, pp. $103-112$.

[9] T. Spyropoulos, K. Psounis and C. S. Raghavendra, C. S, "Spray and wait: an efficient routing scheme for intermittently connected mobile networks", In Proceedings of the 2005 ACM SIGCOMM workshop on Delay-tolerant networking, (2005); ACM, pp. 252-259.

[10] P. Tennent, M. Hall, B. Brown, M. Chalmers and S. Sherwood, "Three applications for mobile epidemic algorithms", In Proceedings of the 7th international conference on Human computer interaction with mobile devices \& services, (2005); ACM, pp. 223-226.

[11] D. Wang, Q. Zhang and J. Liu, "Partial network coding: Concept, performance,and application for continuous data collection in sensor networks", ACM Transactions on Sensor Networks (TOSN),vol.4, no. 3, (2008), pp.14.

[12] J. Widmer and J.-Y. Le Boudec, "Network coding for efficient communication in extreme networks", In Proceedings of the 2005 ACM SIGCOMM workshop on Delay tolerant networking, (2005); ACM, pp. 284-291.

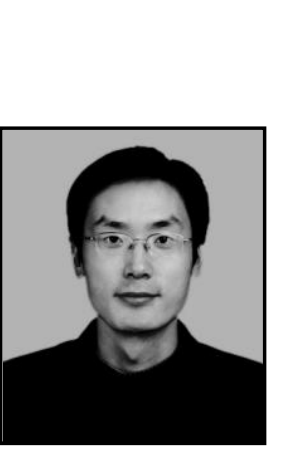

\begin{abstract}
Authors
Hongfeng Wang, he received the M.E. degree in computer application technology from HuaZhong University of Science and Technology of China in 2011. Currently, he is an university lecturer in the School of Computer Science and Technology at Zhoukou Normal University. His research interests include distributed computing, network management and evolutionary algorithm.
\end{abstract}

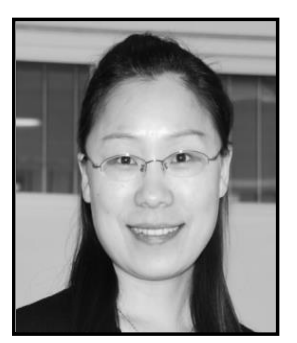

Dingding Zhou, she received the Computer Science degree in School of Computer and Information Engineering from Henan University in 2003.nowadays she is lecturer in Zhoukou Normal University.Her major research interests are in high speed communications, mobility, security, wireless sensor network and QoS guarantees. She is a member of $\mathrm{CCF}(\mathrm{China}$ Computer Federation) in China.

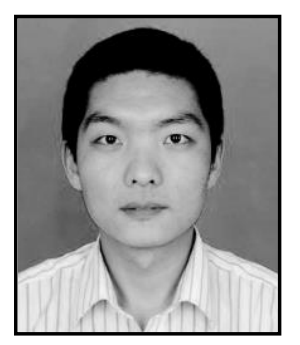

Shi Dong, he received M.S in Computer science from University of Electronic and technology of China.in 2009,Ph.D in computer science from Southeast University. He is currently an associate Professor in the School of Computer Science and Technology at Zhoukou Normal University, and he worked as post doctor researcher in Huazhong University of Science and Technology. His major interests are network management, network security. 
International Journal of Future Generation Communication and Networking Vol. 8, No. 3 (2015) 